\title{
Multimodal Artifact Metrics for Valuable Resin Card
}

\author{
Masaki Fujikawa \\ Kogakuin University, 1-24-2 \\ Nishi-shinjyuku, Shinjyuku, 163- \\ 8677, JAPAN
}

\author{
Kouki Jitsukawa \\ Kogakuin University, 1-24-2 \\ Nishi-shinjyuku, Shinjyuku, 163- \\ 8677, JAPAN
}

\author{
Shingo Fuchi \\ Aoyama Gakuin University, \\ Fuchinobe 5-10-1, Chuo-ku, \\ Sagamihara-city, Kanagawa-Pref., \\ 252-5258, JAPAN
}

\begin{abstract}
This study focuses on multimodal artifact metrics and proposes a technique based on multimodal biometric systems that are a type of biometric identification systems. It is expected that this technique can aid in verifying the authenticity of each artifact in a more accurate manner and in increasing the level of difficulty involved in counterfeiting when compared to those of existing artifact metric techniques. This technique will ensure that artifacts will possess specific characteristics (two or more) that are extracted from different physical characteristics. The study created card-shaped samples with two physical characteristics (electrical and optical characteristics) to prove the feasibility of the proposed technique. The results indicate that two information features, namely sheet resistance and visible light image, which are extracted from the above characteristics, are different in each sample. This indicates that the two information features can correspond to the characteristic information necessary to distinguish each sample.
\end{abstract}

\section{INTRODUCTION}

\section{A. Background and target}

$\mathrm{A}^{\mathrm{s}}$ LTHOUGH the use of artifact metrics is a technique for verifying the authenticity of the artifacts produced daily by manufacturers, its concept is the same as that of biometrics. In artifact metrics, authenticity is verified using characteristic information extracted from an individual artifact. Similarly, biometrics identifies a person using bioinformation extracted from an individual.

It can be said that the word "artifact metrics" stems from a word "biometrics." In biometrics, single modal techniques (methods that use a single type of bio-information to identify individuals) have been prevalent so far; however, multimodal techniques (methods that use multiple types of bio-information to identify individuals) have become common in recent years in order to identify a person with high accuracy and strengthen the robustness of the technique to impersonation attacks. In fact, a technical report on multimodal identification systems was published by ISO [1], and in India, faces, fingerprints, and iris information are

This work was supported by the Kurata Memorial Hitach Science and Technology Foundation and the JSPS KAKENHI Grant Number JP16H07178

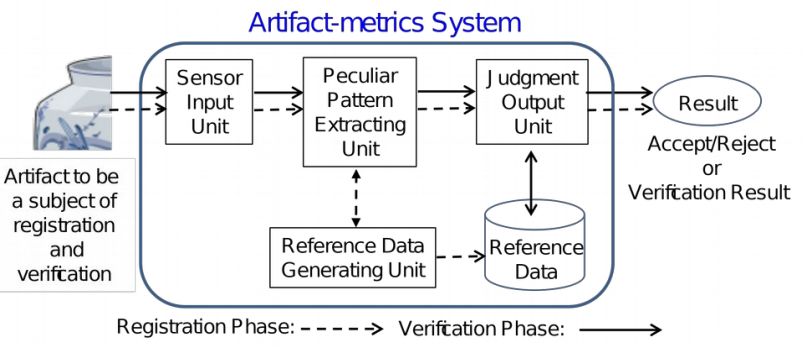

Fig. 1 An overview of the artifact metrics system.

used to identify individuals in the national identification number project [2].

Nowadays, a massive number of copied products that mimic genuine ones have been distributed. Hence, techniques for verifying the authenticity of artifacts with high accuracy and making counterfeiting more difficult are becoming increasingly necessary in manufacturing industries. Therefore, in this paper, we propose a technique that uses "multimodal artifact metrics," which are based on multimodal biometrics. This technique gives artifacts multiple types of characteristic information with different physical characteristics and can verify the authenticity of each artifact using this information.

This paper describes the following contents: In section 2, we describe our technique's concept, prerequisites, and the artifacts targeted in this paper. In section 3, we introduce our approach. In section 4, we explain our experiments of verifying the effectiveness of our method and show its results. Authors describe the considerations for practical use in section 5 and conclude this paper in section 6 .

\section{B. Overview of artifact metrics}

In this section, we describe how characteristic information extracted from artifacts is used in artifact metrics. In artifact metrics, an artifact's unique characteristic information is extracted using sensing devices. This information is embedded in the artifact using the simple methods of adding materials (fillers) with one physical characteristic during the manufacturing process. The particles of the added fillers are distributed randomly and non-uniformly and fixed in the artifact. Their degree of 
distribution reflects the characteristic information. Table 1 shows the physical characteristics of fillers added in the manufacturing process and the characteristic information extracted from them.

Fig. 1 shows an overview of the system (artifact metric system) that uses the artifact metrics. Indicated two phases are almost the same as those of biometrics. In this system, characteristic information is extracted from each artifact before it is shipped and the information is stored in a secure database. To verify the authenticity of an artifact, the system extracts the characteristic information from it and compares this information with the registered feature information from the secure database.

\section{Related studies}

In this section, authors introduce related studies in order to clarify our study's position. Characteristic information extracted from artifacts can be changed depending on the environmental circumstances during extraction (such as temperature, humidity, and position of the artifacts relative to the sensing devices). However, even in such situations, the artifact metric system should be able to verify authenticity stably and correctly based on the strong correlation between the characteristic information registered in the database and the information features extracted during verification.

There is an approach to increase the number of characteristic information in order to find strong correlations between both registered and extracted information. A method was proposed to extract two characteristic information from a filler with one physical feature [3]. In this method, a filler with an optical feature (glass phosphor powder) is mixed with paint and glaze and adhered onto the surface of porcelain in order to bond the particles of the filler onto the ceramics during the firing process. There is a difference between the density of light-emitting ions and the particle diameters that depends on the observation points. The method [3] utilizes the difference between the distributions of the light-emitting spectrum and lightemitting intensity that is caused by this difference and uses these two distributions as characteristic information.

\section{II.MULTIMODAL ARTIFACT METRICS}

\section{A. Concept}

The idea for the technique proposed in this paper (multimodal artifact metrics) is based on multimodal identification in biometrics. However, unlike previous study [3], as described in Section 1.3, this technique gives an artifact two or more physical characteristics and extracts two or more types of characteristic information from them. The main difference between our method and that of [3] (i.e., the advantage of our method) is that it is able to increase the number of characteristic information that can be extracted from the artifact. This is because the previous study gives the artifact only one physical characteristic so that clearly fewer number of characteristic information can be extracted

TABLE I.

PHYSICAL FEATURES AND EXTRACTED CHARACTERISTIC INFORMATION

\begin{tabular}{c|l}
\hline $\begin{array}{c}\text { Physical } \\
\text { characteristics }\end{array}$ & \multicolumn{1}{c}{ Extracted feature information } \\
\hline $\begin{array}{c}\text { Optical } \\
\text { characteristics }\end{array}$ & $\begin{array}{l}\text { Particles' optical characteristics (reflection, } \\
\text { transmission, infraction, and fluorescence) and their } \\
\text { degree of distribution reflect the characteristic } \\
\text { information, which is extracted by sensors that can } \\
\text { detect light intensity }\end{array}$ \\
\hline $\begin{array}{c}\text { Magnetic } \\
\text { characteristics }\end{array}$ & $\begin{array}{l}\text { Particles' magnetic characteristics (attraction and } \\
\text { repulsive force) and their degree of distribution } \\
\text { reflect the characteristic information, which is } \\
\text { extracted by sensors that can detect a change in } \\
\text { magnetism. }\end{array}$ \\
\hline $\begin{array}{c}\text { Electrical } \\
\text { characteristics }\end{array}$ & $\begin{array}{l}\text { Particles' electric characteristics (electrical charge) } \\
\text { and the degree of distribution reflect the } \\
\text { characteristic information, which is extracted by } \\
\text { sensors that can detect the quantity of electric charge. }\end{array}$ \\
\hline $\begin{array}{c}\text { Vibration } \\
\text { characteristics }\end{array}$ & $\begin{array}{l}\text { Particles' vibration characteristics (sonic waves) and } \\
\text { the degree of distribution reflect the characteristic } \\
\text { information, which is extracted by sensors that can } \\
\text { detect sonic waves. }\end{array}$ \\
\hline
\end{tabular}

TABLE II.

CATEGORIZATION OF SYNTHETIC RESIN PRODUCTS

\begin{tabular}{|c|c|}
\hline \multirow{3}{*}{ Type 1} & $\begin{array}{l}\text { Definition: } \\
\text { The majority of the artifact is formed of synthetic resin. }\end{array}$ \\
\hline & $\begin{array}{l}\text { Usage } 1: \\
\text { Credit cards }[4] \text { Cash cards }[5] \quad \text { SIM cards, etc. } \\
\text { Usage 2: } \\
\text { Prepaid cards }[6] \quad \text { loyalty cards, etc. } \\
\text { Usage } 3: \\
\text { Exterior finish of home appliances, hardware token (One- } \\
\text { time password generator, etc.) }\end{array}$ \\
\hline & $\begin{array}{l}\text { Material: } \\
\text { PVC is used for Usage 1, PET is used for Usage 2, and ABS } \\
\text { is used for Usage } 3 .\end{array}$ \\
\hline \multirow{3}{*}{ Type 2} & $\begin{array}{l}\text { Definition: } \\
\text { The surface of the artifact is coated or painted by synthetic } \\
\text { resin. }\end{array}$ \\
\hline & $\begin{array}{l}\text { Usage 1: } \\
\text { Products made of ABS or polycarbonate without a baked } \\
\text { finish (e.g., wrist watches[7] and spectacle frames for } \\
\text { eyeglasses). This finish requires more than } 150^{\circ} \mathrm{C} \text {. } \\
\text { Usage 2: } \\
\text { Products made of aluminum or brass without a baked finish } \\
\text { (e.g., cast aluminum wheels[8]). }\end{array}$ \\
\hline & $\begin{array}{l}\text { Material: } \\
\text { Acrylate resin, urethane resin, fluorine resin, or epoxy resin } \\
\text { is used for coating or painting in both usages. }\end{array}$ \\
\hline
\end{tabular}

from the artifact, whereas our method gives the artifact two or more physical features.

Our method has two contributions to artifact metrics technology. One is the ability to verify the authenticity stably and correctly (as we mentioned in the previous section), as our method can find a strong correlation between the characteristic information registered in a 
database and the feature information extracted from the artifact by increasing the number of characteristic informati-

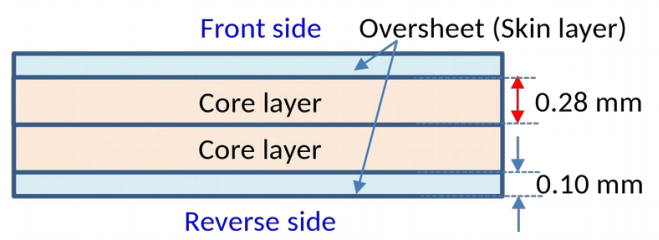

Fig. 2 A cross section of a valuable card.

on contained in artifact.Another one is to heighten the difficulty of counterfeiting for forgers, as the number of characteristic information contained in genuine products is increased.

\section{B. Application to valuable card made from synthetic resin}

Although we are still investigating artifacts that are appropriate for multimodal metrics, we focus here on artifacts made from synthetic resin as one application in order to verify the applicability of our method.

We have two reasons for focusing on this type of artifact. One is that such artifacts are common in our daily life. In general, synthetic resin is easy to form and resists acid and alkaline corrosion. Furthermore, it is used to add features and characteristics to products in order to make them fit for use. Although it has the reputation of being non-conductive, flammable, and non-biodegradable, new types of synthetic resin with electro-conductive, flame-retardant, and biodegradable features have been developed. It can also be recycled.

Another reason is the existence of copied synthetic resin products. Table 2 categorizes synthetic resin products. Products for which the existence of copied products have been confirmed are underlined and the reference given. (In Table 2, products are categorized into two groups, and the definition, purpose of use, and specific name of the synthetic resin are indicated.) We predict that security devices (such as SIM cards and hardware tokens which are categorized in Type 1) would be copied in the near future.

Specifically, the present study focuses on valuable cards composed of synthetic resin (such as credit cards) that are widely used worldwide. Additionally, a technical approach is also proposed to provide characteristic information with respect to the products and to verify the applicability of the proposed method through experiments.

\section{Requirements}

This section lists four requirements that must be satisfied by the technical approach in which multimodal artifact metrics are applied to valuable cards.

Requirement 1: Characteristic information can be formed on the print surface of valuable cards.

It is desirable for the technical approach to form characteristic information on the print surface of valuable cards such that the printing of card information and the formation of characteristic information can be performed on the same surface. This includes the advantages of simultaneously reading and extracting the two abovementioned pieces of information. As a reference, valuable cards are formed by four resin layers (two core layers and two skin layers) $[9,10]$ as shown in Fig. 2, and the characters and images printed on the surface of core layer are protected by a skin layer.

Requirement 2: Characteristic information is not affected by temperature.

Valuable cards are used in a situation characterized by a wide range of temperature. Hence, it is necessary that temperature should not affect the characteristic information formed on the surface of the core layer.

Requirement 3: Valuable cards should be tamper-resistant. Valuable cards should possess a tamper-resistance function to ensure the difficulty involved in copying the characteristic information. With respect to concrete, the characteristic information breaks easily if the skin layer is stripped.

Requirement 4: Materials used to form characteristic information are safe.

Materials used to form valuable cards (such as synthetic resin and the paint) are associated with a low risk of affecting human skin. Hence, it is necessary that the material used to form characteristic information on the surface of core layer should possess the same feature.

\section{Preconditions}

We set the prerequisites shown below in order to clarify our discussion.

Condition 1: The goal of this study involved verifying the applicability of the proposed approach to apply multimodal artifact metrics to valuable cards. In the case of concrete, three objectives are involved: (1) Creating samples with two characteristic information features (derived from different physical characteristics) on the surface of resin plate that is used in a manner similar to a real core layer; (2) Extracting two types of characteristic information from samples; (3) Confirming the differences in the types of characteristic information extracted from each sample.

Condition 2: The goal of this paper is to determine whether we can apply multimodal artifact metrics to samples made of synthetic resin (conductive polymer). Hence, the implementation of multimodal artifact metrics (the construction of an artifact metric system and its evaluation) is not within the scope of this paper. 


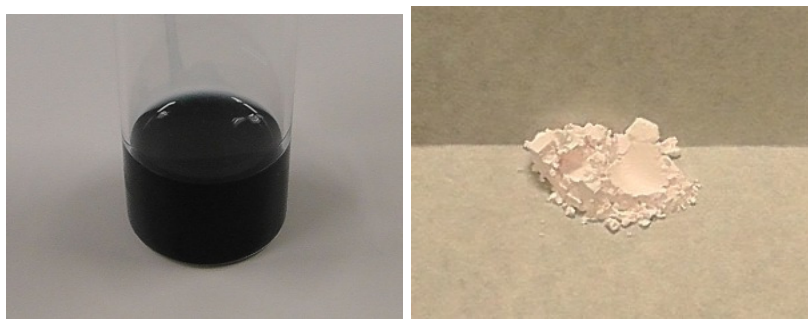

Fig. 3 Conductive polymer paint (left) and IR up-conversion phosphor (right).

Front side

Oversheet (Skin layer)

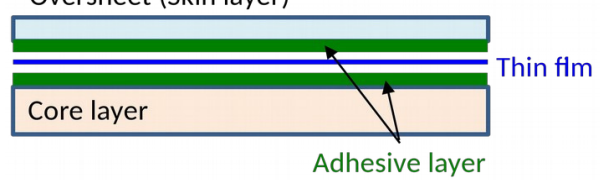

Fig. 4 Thin film and adhesive layers.

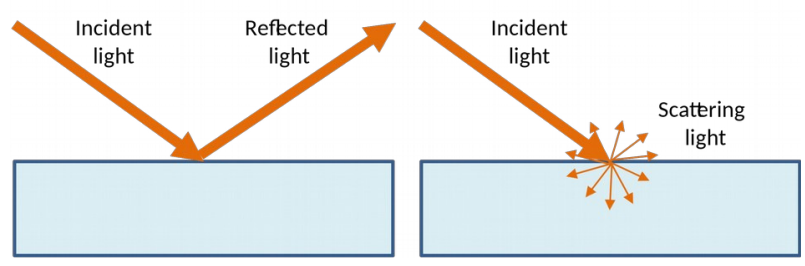

Fig. 5 Reflection and scattering.

\section{APPROACH}

\section{A. Forming characteristic information}

To satisfy four requirements described in section 2.3 , it is necessary to form a thin film on the surface of the core layer. In this paper, we use a mixture of conductive polymer paint and small amount of infrared up-conversion phosphor powder (hereinafter referred to as IR phosphor powder). The conductive polymer paint (as shown in Fig. 3) possesses low electrical resistance and is used as a material to create a transparent electrode. It also possesses the three features, namely the conductivity is not affected by temperature [11], there is a low risk of affecting human skin, and it is more flexible and possesses high transparency when compared with that of Indium Tin Oxide used as a material for making transparent electrodes. The transparent electrode formed by the polymer paint is not broken, and its electrical resistance (sheet resistance) is not changed by the bending force. However, it is easily broken by a physical force such as scratching. Conversely, this leads to the realization of tamper-resistance for the characteristic information. For example, as shown in Fig. 4, a tamper-resistance function is realized by sandwiching the thin film with a hightransparent adhesive allocated to the core as well as the skin layer. If the skin layer is peeled off, then the characteristic information included in the thin layer will be broken such that it is not possible for a counterfeiter to easily analyze the film.
The IR phosphor powder (as shown in Fig. 3 with a particle diameter of approximately $2-3 \mu \mathrm{m}$ ) is a nonconductive substance. The optical characteristics are not affected by temperature and the toxicity is significantly lower than that of other phosphors that emit visible light (e.g., quantum dot semiconductor phosphor). The IR phosphor powder emits visible light with a peak wavelength of $\mathrm{X}$ by optical excitation with a peak wavelength of $\mathrm{Y}$. Both peak wavelengths can be changed by adjusting the composition of the IR phosphor powders. Additionally, due to ease of handling, IR phosphor is widely used for bioimaging, tracking, and simple authentication of products.

\section{B. Reasons for selecting a thin film to form characteristic information}

This section describes the reasons for adopting the thin film instead of the core layer as the location to form characteristic information. The core layer of valuable cards is composed of PVC or polyvinyl chloride. Specifically, PVC is a non-conductive substance and does not possess distinguishing optical and electrical characteristics, and thus it is necessary to add a filler with two physical characteristics in PVC. Incidentally, the preliminary experiment indicates that it is possible to induce electrical characteristic in a non-conductive synthetic resin (epoxy). However, it is not appropriate to consider the extracted resistance as a type of characteristic information because the resistance is very high and not stable (with a value between $4.0 \Omega$ and $6.0 \times 10^{12} \Omega$ ) [12]. This indicates that the same situation could occur in PVC.

The optical characteristic is also considered. A large part of light irradiated to the core layer reflects on its surface such that it is hard for scattering to occur due to the high glossiness of the PVC (as shown in Fig. 5). Hence, the amount of optical characteristic information is low since the luminescence is observed in a 2-dimensional surface and not in a 3-dimensional manner even if the IR phosphor powder is added to PVC.

Conversely, in the proposed approach, observed electrical resistance is likely to be low and stable since a major part of the thin film is dominated by a conductive substance. Additionally, reproducibility (this implies that the same resistance is observed throughout at the same point) can be observed if the resistance is stable. Hence, it is possible that the resistance extracted from the thin film can correspond to the electrical feature information.

This is followed by examining the optical characteristic. The glossiness of the thin film formed by the conductive polymer is significantly lower than that of the PVC, and thus the light irradiated to the thin film scatters on its surface (as shown in Fig. 5). Hence, the IR phosphor that exists both at the surface and inside the thin film is excited, and the luminescence of IR phosphor can be captured 3dimensionally by a camera (this means that the amount of optical feature information increases). Thus, the thin film is 


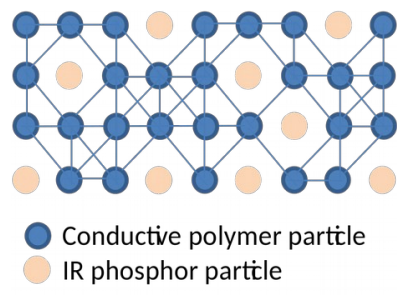

Fig. 6 An illustration depicting densities of the bonded molecules.

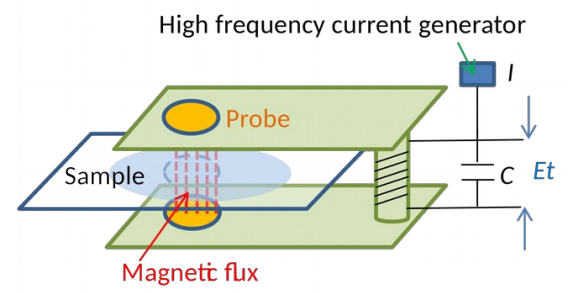

Fig. 7 An overview of the eddy current measuring method and its electrical circuit.

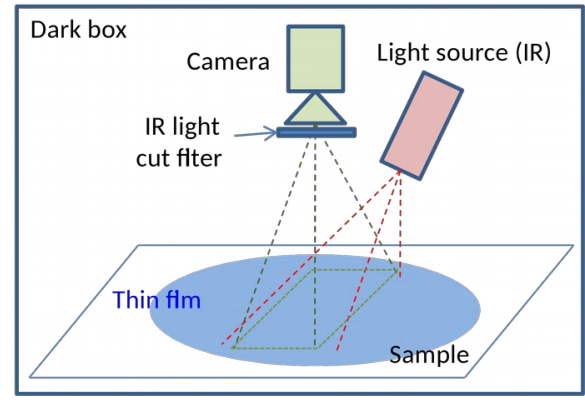

Fig. 8 Photo shooting method.

adopted as the location for the formation of characteristic information because of the fore-mentioned reasons.

\section{Forming information features}

This section describes the manner in which the two types of information features are formed by using a mixture of a conductive polymer and IR phosphor powder as described in section 3.1. The thin film is formed when the moisture evaporates after a thin coat of the liquid mixture is painted on the surface of core layer. Thus, the connections between the particles of the polymer are variable because they spread randomly and non-uniformly due to the existence of IR phosphor particles when the thin film is formed (as shown in Fig. 6). Hence, it is possible to extract electrical resistance as an information feature because it is reflected by the above connection. Furthermore, the size of each IR phosphor particle (it is reflected as light emission intensity), the degree of reflection, transmission, and inflection of the light, and the degree of dispersion of the phosphor particles are also simultaneously determined in the thin film. Hence, they are reflected as the characteristic information and extracted as an information feature by capturing images using a camera with optical excitation. The proposed approach that extracts two types of characteristic information without contact is applied. Hence, this can avoid the risk of scratching the surface of valuable cards while extracting characteristic information.

\section{Extraction of characteristic information}

First, the extraction method of electrical resistance (sheet resistance) is described. In general, probes are used to measure electrical resistance of samples and there are two types of methods of using probes (two-terminals and fourterminals measuring method). On the other hand, in order to measure the resistance of thin film (or the sheet resistance), there is a method not to touch any probes to the samples (it is called eddy current measuring method). In this method, as shown in Fig. 7, sample is set between the gap of the probe. Measuring procedure of the surface resistivity is shown below:

(1) Generate magnetic flux by adding high frequency between the probe.

(2) Eddy current occurs in the sample when sample is inserted between the gap of the probe. At this time, electrical power loss occurs as the current is consumed in the sample. The current in the circuit is also decreased in proportion to the above loss.

(3) As decreased current value is inversely proportional to the sample's resistance, surface resistivity is calculated by using these values and the thickness of the sample.

The fore-mentioned measurement method appears as appropriate since it is easy to break the thin film composed of a conductive polymer when it is subject to a physical force (e.g., scratching). In the experiment (refer section 4), the difference between the sheet resistances extracted from samples is then confirmed.

This is followed by describing the shooting method of the luminescence of the IR phosphor caused by an optical excitation by using a camera. As shown in Fig. 8, the photographing devices and the sample are set in the dark box. This is because the aim includes capturing the visible light components only from the luminescence by using an image sensor of the camera. The camera faces the sample, and IR light is irradiated on the sample surface. The particles of the IR phosphor emit visible light with a specific peak wavelength by optical excitation, and the scene is captured by using the camera. This is followed by confirming the differences between each image obtained from the samples (refer section 4.)

\section{E. Verification of authenticity}

Similar to other techniques proposed for artifact metrics so far, authenticity verification is done by calculating the degree of similarity between the information features registered in a secure database beforehand and those extracted during verification. As described in section 2.4, the implementation of multimodal artifact metrics is beyond the scope of this study because the main aim of this study involves creating samples with two types of characteristic 
information derived from different physical characteristics on the surface of the resin plate, extracting two different types of information features from the samples, and confirming the differences between the extracted information. Hence, in this paper, we refrain from describing how to set the threshold level for calculating the degree of similarity. However, if we implement our technique, we should carefully calculate the degree of similarity in order to avoid incorrectly determining genuine products as copied products and vice versa [3].

\section{EXPERIMENTS}

\section{A. Appropriate amount of IR phosphor powder}

Generally, the decrease in moldability and the decline in strength can be observed if there is an increase in the amount of fillers added to the synthetic resin [13, 14]. Conversely, it is not possible to extract sufficient characteristic information if a low amount of fillers is used. Hence, to determine the appropriate amount of IR phosphor powder to be added to the conductive polymer, four types of mixtures with different weight ratios of phosphor powder $(5 \%, 10 \%, 20 \%$, and $40 \%)$ are created. The liquid mixture is then used to confirm whether or not the thin film is formed on the surface of the resin plate that is used to resemble the real core layer.

A polypropylene plate with hydrophilic characteristics is used. The mixture of liquid spreads in a circle on the surface of the plate and corresponds to a thin film state due to hydrophilic features of the plate and the gravity following the infusion of $5 \mathrm{ml}$ of liquid by using a syringe. The mixture is kept undisturbed for $60 \mathrm{~min}$ to evaporate moisture from the liquid, and a transparent blue thin film is formed from $5 \%$ and $10 \%$ mixture liquid. The film does not form from $20 \%$ and $40 \%$ mixture liquid (as shown in Fig. 9). However, the existence of the IR phosphor powder is clearly observed. This phenomenon corresponds to that observed in previous studies $[13,14]$.

It is desirable for the synthetic resin to possess a smaller amount of fillers. Hence, it is determined hereinafter that a $5 \%$ weight ratio of IR phosphor powder is necessary to form the mixture with a conductive polymer. There are several methods to create a thinner film including the spin-coating method and the dip-coating method. However, these methods are not used as the aim of this study involves verifying the applicability of the proposed approach.

\section{B. Sample making}

It is necessary for the sample thickness to range between $0.5 \mathrm{~mm}$ to $1.5 \mathrm{~mm}$ and for the film diameter to exceed the probe diameter when the sheet resistance of the thin film is measured. In the experiment, the sheet resistance meter that includes a probe with a diameter of $14 \mathrm{~mm}$ is used, and thus a transparent polypropylene plate with a thickness of 0.5 $\mathrm{mm}$ is used to resemble the real core and skin layer. The thin film with a diameter exceeding $14 \mathrm{~mm}$ is used on the surface of the core layer, and the film is sandwiched by the core and the skin layer. A total of 20 samples are created, and each sample has a thin film and two polypropylene layers.

\section{Extraction of feature information (sheet resistance)}

As shown in Fig. 7, an experiment is performed to confirm the differences in the sheet resistance between samples. Fig. 10 shows the sheet resistance extracted from the central part of the samples. The vertical axis shows the sheet resistance (ohm/square) and the horizontal axis shows the sample number. The results indicate that there are differences in the sheet resistance between the samples and that the same resistance can be extracted from same point even when there are differences between the insertion angles of the sample and the probe (this implies that the result is replicable).
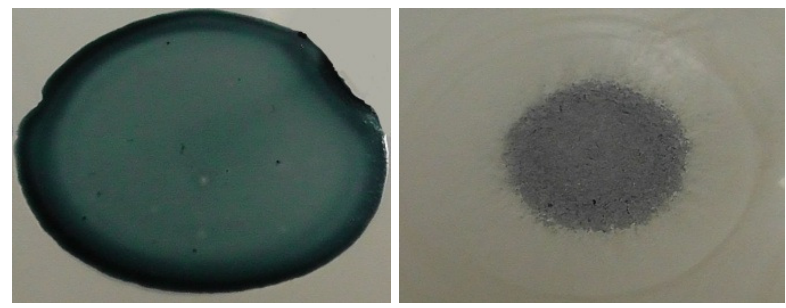

Fig. 9 Formed thin film (Left: 5\%, Right: 20\%).

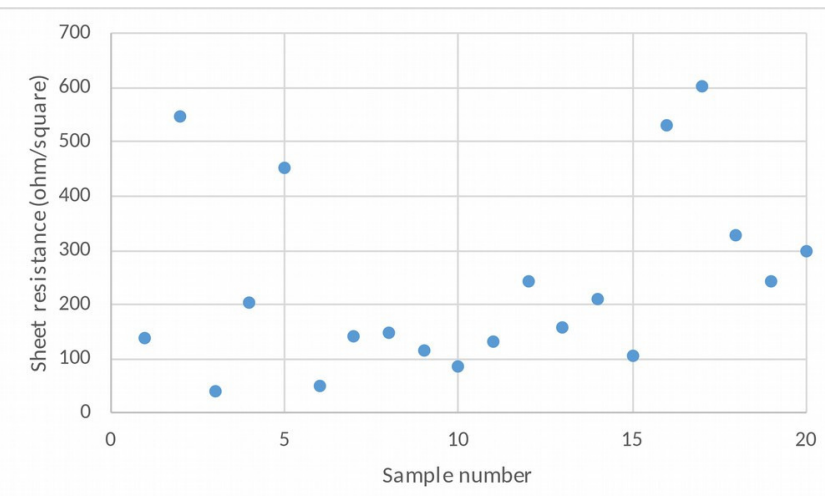

Fig. 10 Sheet resistance (Weight percentage 5\%).

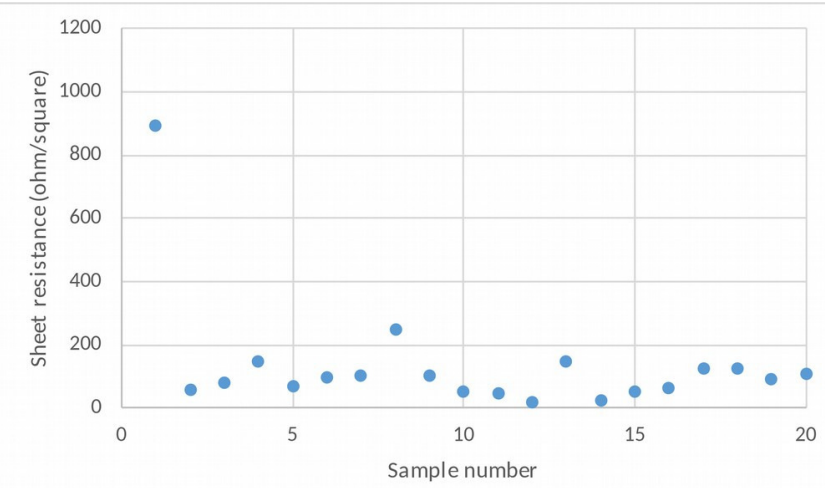

Fig. 11 Sheet resistance (without IR phosphor powder). 


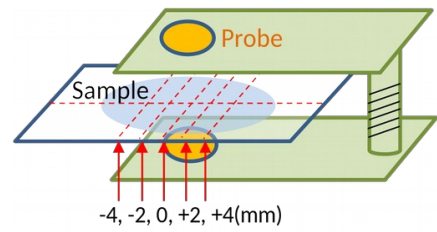

Fig. 12 Measurement of sheet resistance.

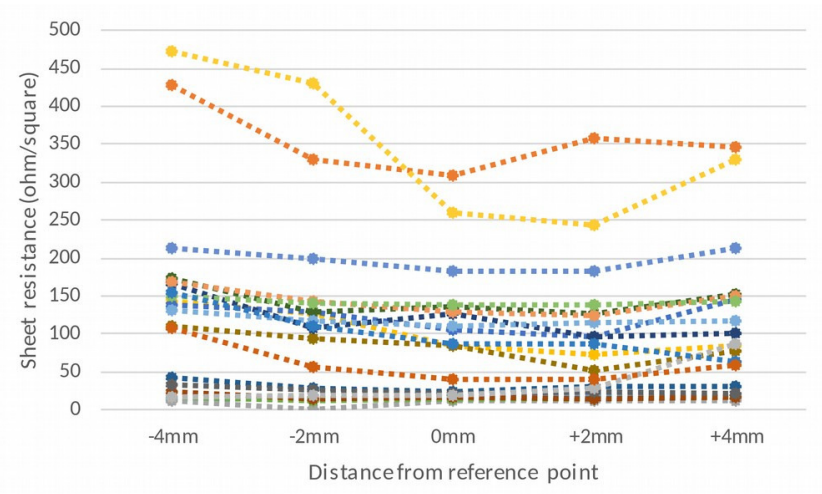

Fig. 13 Sheet resistance (Weight percentage 5\%).

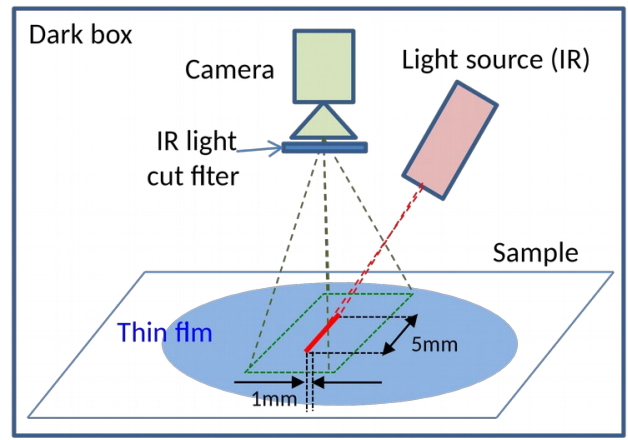

Fig. 14 Experimental system.


Fig. 15 Sample images (Weight percentage 5\%).
As a reference, Fig. 11 shows the sheet resistance extracted from the central part of the samples without IR phosphor powder. The degree of variation in the samples is less than that shown in Fig. 10 (where Sample number 1 exhibits high sheet resistance. It is assumed that the tiny solid pieces contained in the conductive polymer paint probably behaved as the resistance and affected the eddy current).

As shown in Fig. 12, the sheet resistance is measured at each point when the sample is moved by $2 \mathrm{~mm}$ from the reference point. Fig. 13 shows the line graph. The vertical axis denotes the sheet resistance (ohm/square), the horizontal axis denotes the distance from the reference point, and each line denotes the samples. The results indicate the difference in the sheet resistance from different samples, and the same resistance could be extracted from same point on the sample even if there are differences in the insertion angle between the sample and the probe (this implies that the result was replicable). The findings also reveal that there are differences in sheet resistances within the same sample albeit extracted from different observation points. Therefore, it is assumed that the sheet resistance is potentially a type of characteristic information that can be used to authenticate valuable cards.

\section{Extraction of feature information (visible light images)}

As shown in Fig. 8, another experiment is conducted to verify two characteristics. The first characteristic involves observing the visible light emission from IR phosphor contained in samples with peak wavelengths of $548 \mathrm{~nm}$ and $554 \mathrm{~nm}$ by optical excitation with a peak wavelength of 980 $\mathrm{nm}$. The second characteristic involves observing the differences in the luminescence states in each sample. We made experimental system shown in Fig. 14 and irradiated IR light (width: approx. 1mm, length: approx. $5 \mathrm{~mm}$ ) at the center of the surface of samples. Fig. 15 shows six images obtained from 20 samples (the bottom right corner shows the sample number). The luminescence of the IR phosphor particles is expressed as whitish spots and the differences in the emission intensities and the degree of the dispersion of phosphors can be observed from the images. Hence, it is assumed that the visible light images can potentially correspond to a type of characteristic information that is used to distinguish valuable cards.

\section{V.CONSIDERATIONS}

This section considers the requirements that satisfy the proposed approach and the difficulty of counterfeiting feature information. The section also discusses the feasibility of the proposed method.

\section{A. Satisfaction of the requirements}

This section describes the manner in which the proposed approach satisfies the requirements listed in section 2.3. First, with respect to Requirement 1, the proposed approach forms a thin film on the surface of the core layer and provides two types of characteristic information with respect 
to the film. In the experiment, the thin film is formed on the surface of the polypropylene plate used to resemble the real core layer and to extract two types of characteristic information from it. It is possible to perform printing on the surface of the polypropylene in a manner similar to other types of synthetic resins such as polyethylene, polyvinyl chloride, and polystyrene. Hence, it is assume that the approach satisfies Requirement 1.

This is followed by considering Requirement 2 . It is possible to use both materials (the conductive polymer paint and the IR phosphor powder) adopted in the proposed approach across a wide range of temperatures since the materials are not affected by temperature. Additionally, mixing the above materials does not produce any substances that are susceptible to temperature. Hence, it is assumed that the approach satisfies Requirement 2.

With respect to Requirement 3, although the thin film composed of the conductive polymer is flexible, it breaks easily when subject to physical force (such as light scrubbing on the surface of the thin film by using a finger). Therefore, it is assumed that a tamper-resistant feature can be realized by sandwiching the thin film between the core and the skin layer with adhesive (see Fig. 4). Hence, it is assumed that the proposed approach satisfies Requirement 3.

With respect to Requirement 4, a conductive polymer does not contain any toxic elements and uses safe solvents such as water and ethanol. Similarly, the IR phosphor is not harmful since it is a stable oxide. An example of a stable oxide is lead glass in which lead glass is not poisonous although lead is toxic, and thus it is used as a material to create fine glass tableware. Hence, the synthetic resin and filler used in the proposed method do not pose any risks to human skin, and it is assumed that the approach satisfies Requirement 4.

\section{B. Difficulty of counterfeiting information features}

This section describes the difficulty of counterfeiting two types of feature information. First, the sheet resistance is considered. As shown in Fig. 10 and Fig. 13, there are differences in the sheet resistances extracted from samples. Additionally, differences in sheet resistances are observed at each observation point located in the same sample. A reason for these differences could be attributed to the dispersion of the non-conductive IR phosphor particles that are spontaneously and randomly scattered in the conductive polymer paint.

This implies that the density of IR phosphor particle does not form evenly. To counterfeit a genuine sample to create a fake sample that has the same sheet resistance as the genuine sample, the following tasks are necessary:

(1) Obtaining materials (polypropylene plate, IR phosphor powder, and conductive polymer paint) used to create a genuine sample A.

(2) To create a thin film with same area, thickness, and density of sample A, it is necessary to compose a

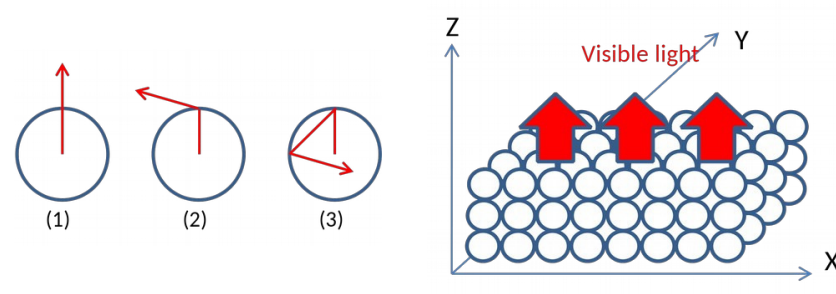

Fig. 16 Light paths (left) and counterfeiting method (right).

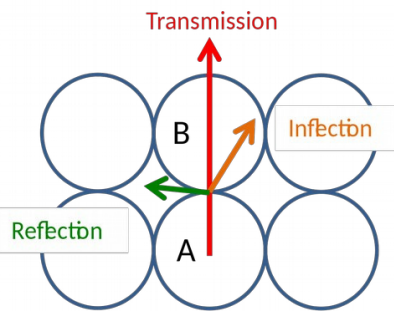

Fig. 17 Inflection, transmission, and reflection at the surface of particle.

mixture of conductive polymer and IR phosphor powder and to drop the mixture on the surface of the polypropylene plate. Next, prior to drying the mixture of liquid and converting it into a thin film, it is necessary to move and fix each IR phosphor particle (with an approximate particle diameter of several micrometers) at the same three-dimensional location wherein the particle from sample A exists in its thin film.

It is relatively simple to perform Step (1) in manufacturing of a genuine sample. However, to perform Step (2), a counterfeiter would need to use "a high resolution microscope" and "a technique to move and fix each particle at the designated three-dimensional location. Each particle exists in the liquid that is gradually cured." Hence, a high level of difficulty is associated with counterfeiting. Additionally, as the size and the shape of each particle are spontaneously and randomly determined, it is extremely difficult to create a fake sample with the same particles as those contained in genuine sample A.

Next, we consider the visible light images. Each sample and camera was faced each other and the samples are set on the optical surface plate, so that the images were shot along the z-axis. The intensity of the visible light emission has a relationship with the density of the IR phosphor particles, and a higher density leads to stronger infrared light emission. In addition, there are three possible light paths for the visible light emitted from the IR phosphor particle (see Fig. 16, left). One is the path directly from the center of particle, the second one is the path after inflection at the particle's boundary, and the last one is the path that does not come out of the particle because of repeated reflection at the particle's boundary. It seems that the sample might be counterfeited if counterfeiters could gather particles with direct light paths, as shown in Fig. 16, left (1) and 
accumulate them on the $\mathrm{z}$-axis as in Fig. 16, right. However, as shown in Fig. 17, visible light emitted out of IR phosphor particle A might have reflection, inflection, and transmission at particle B's boundary, so it is hence quite difficult to emit visible light only along the z-axis as intended by counterfeiters (in fact, counterfeiting is quite difficult as the shape of the particles is not spherical). Hence, we conclude that it is difficult to counterfeit samples with the same visible light images because of the above considerations.

As previously mentioned, the proposed approach can increase the level of difficulty involved in counterfeiting.

\section{Application of the Phase-Only Correlation method}

In a biometric system, it is difficult to observe reproducibility in images involving scans of fingerprints, iris, and retina, and differences (such as slight parallel translation, rotation movement, and brightness) are observed between the fore-mentioned images. Hence, an image matching technique termed as Phase-Only Correlation (POC) is adopted [15] to stably evaluate the similarity in images despite the existence of the fore-mentioned differences. This system corrects the angle and the position displacement and extracts common areas between the registered image and the image obtained in the verification phase. This is followed by calculating the degree of correlation degree and deriving the similarity. The results indicate that the proposed algorithm is more robust than algorithms proposed by previous studies with respect to differences in a photo shooting environment.

We will conduct an experiment in order to confirm the applicability of this method with visible images used in our approach. In concrete, we change the intensity of excitation light and the location of samples and shoot visible images while having optical excitation. We then confirm the degree of similarity in each sample by using obtained images.

\section{Consideration of forming thin film}

In the experiment, a liquid mixture consisting of the conductive polymer and the IR phosphor powder is dropped by using a syringe to create a thin film by employing gravity in conjunction with the hydrophilic features of the resin plate. Although the thickness is low, the film is opaque (as shown in Fig. 9 (left)) as it is much thicker than the transparent electrodes composed of conductive polymer paint.

To avoid affecting the visibility of characters and images printed on the surface of the real core layer, it is necessary to form a thin layer with a thickness equal to that of the transparent electrodes on the entire surface of the real core layer. This is because the visibility of printed characters and images could be affected by angularities generated at the boundary between the thin film and the core layer if the thin film is formed on the part of the surface of the core layer.

In contrast to the dip-coating method, the spin-coating method described in section 4.1 has an advantage as it forms a thin film with a small amount of coating liquid [16]. Additionally, other spin-coating methods, such as using conductive polymer paint as a coating liquid and its application to the surface of the synthetic resin plate, were proposed by extant studies. Therefore, it is assumed that the proposed approach (forming a thin layer on the surface of the core layer) is associated with high feasibility. A future study will confirm two objectives, namely forming a thin film on the surface of the core layer by using a liquid mixture and the spin-coating method and extracting two information features from a thin film without contact.

\section{CONCLUSION}

In this paper, we proposed multimodal artifact metrics in order to authenticate an artifact with high accuracy and heighten the difficulty of counterfeiting. This technique is based on multimodal identification in biometrics. This method can give two or more information features generated from two or more physical characteristics into an artifact and verify the authenticity of each artifact using the extracted information.

The study focused on valuable cards composed of synthetic resin as artifacts for the application of multimodal artifact metrics because the existence of copied cards was reported by extant studies. Additionally, an experiment was conducted to confirm the applicability of the proposed approach (forming characteristic information on the surface of the core layer). In the experiment, a thin film was formed on the surface of polypropylene plate by using conductive polymer paint and an IR phosphor powder. Samples consisting of a thin film and two polypropylene plates were composed in which the thin film was sandwiched by the plates. Two types of information features, namely sheet resistance and visible images, were extracted from samples without contact. The result of the experiments indicated that there were differences in the information extracted from each sample, and thus there is a high possibility that this can be used as feature information. Furthermore, the proposed approach satisfied four requirements and demonstrated that it increased the difficulty of counterfeiting.

To implement the approach stated in this study, a future study will involve developing a visible image matching algorithm by using a POC method and confirming the feasibility of forming a thin film on the surface of the core layer. Thus, future studies will explore developing the proposed multimodal artifact metrics and related techniques.

\section{REFERENCES}

[1] ISO/IEC TR 24722:2015, "Information technology -- Biometrics -Multimodal and other multibiometric fusion," December 2015

[2] Arvind Slwal, Sunil Kumar Gupta, Surender, and Anubhuti, "Template security analysis of multimodal biometric frameworks based on fingerprint and hand geometry," Perspectives in Science, Vol. 8, pp. 705-708, Sep. 2016. https://doi.org/10.1016/j.pisc.2016.06.065

[3] Masaki Fujikawa, Fumihiko Oda, Kengo Moriyasu, Shingo Fuchi, and Yoshikazu Takeda, "Development of the New Artifact-metrics Technology for Valuable Pottery and Porcelain products," Journal of Information Processing Society of Japan, Vol. 55, No. 9, pp. 19922007, Sep. 2014 
[4] Europol, "International credit card fraud syndicate active in Europe and Asia disrupted," July 8th 2016, available: https://www.europol.europa.eu/content/international-credit-cardfraud-syndicate-active-europe-and-asia-disrupted

[5] Focus Taiwan, "Taiwanese arrested for using counterfeit bank cards in Bangkok," July 4th 2016, available: http://focustaiwan.tw/news/asoc/201607040020.aspx

[6] CBS Miami, "Father, Son Arrested In \$50K Gift Card Fraud Raid," September 1st 2016, Available: http://miami.cbslocal.com/2016/09/01/counterfeit-credit-cardoperation-busted-in-sw-dade/

[7] CASIO America Inc., "Buyer Beware of Purchases Made From Unauthorized Resellers Of Casio premier G-Shock Products,' Available: http://www.gshock.com/support/Unauthorized

[8] news.com.au, "Tens of thousands of cunterfeit wheels made in China are on Australian roads, experts warn," Available: http://www.news.com.au/finance/business/manufacturing/tens-ofthousands-of-counterfeit-wheels-made-in-china-are-on-australianroads-experts-warn/news-story/bbf2565440a7ddf0c291a7ef9c4e9815

[9] Sutton, Caroline and Kevin Markey, "More How Do They Do That?" New York: William Morrow \& Co., 1993.
[10] ISO ISO/IEC 7810:2003, Identification cards -- Physical characteristics

[11] Akio Taniguchi, "Application of Organic Semiconductors," CMC Technical Library, No. 297, pp. 100/101, CMC publishing. (Japanese)

[12] Masaki Fujikawa, Kouki Jitsukawa, and Shingo Fuchi, "The Proposal for the Multimodal Artifact Metrics and the Study of Applicability to Synthetic Resin Products," Proc. of the Computer Security Symposium, pp. 343-348, Oct. 2016. (Japanese)

[13] Hisanori Shinohara, "Recent Advances in the Research and Development of Nanocarbon Materials," CMC publishing, 2008.

[14] Hiroshi Ishikawa and Yoshihiro Tomioka, "Deterioration of Polymer Material, Journal of the Society of Heating," Air-Conditioning and Sanitary Engineers of Japan, Vol. 79, No. 10, pp.961-968, 2005 (Japanese)

[15] Nabilah Shabrina, Tsuyoshi Issiki, and Hiroaki Kunieda, "Fingerprint authentication on touch sensor using Phase-Only Correlation method," Proc. of the International Conference of Information and Communication Technology for Embedded Systems, May 2016. https://doi.org/10.1109/ICTEmSys.2016.7467127

[16] Hiromitsu Kozuka, "Fundamentals of sol-gel coating technique," New Glass, Vol. 25, No. 3, pp. 40-45, 2010. 